\title{
NANOTECNOLOGIA APLICADA À ONCOLOGIA: UMA PERSPECTIVA SOBRE O TRATAMENTO DO CÂNCER UTILIZANDO ÓXIDO DE GRAFENO ${ }^{1}$
}

\author{
NANOTECHNOLOGY APPLIED TO ONCOLOGY: A PERSPECTIVE ON \\ CANCER TREATMENT USING GRAPHENE OXIDE
}

\section{Lenise Deon Pompeu², Ana Júlia Figueiró Dalcin ${ }^{3}$, Evelin Cogo de Oliveira ${ }^{4}$, Andriele Pinheiro Pinton ${ }^{4}$ e Nara Saraiva Dutra ${ }^{5}$}

\section{RESUMO}

O câncer é causado pela transformação celular no processo de divisão celular. As células cancerosas geralmente são descontroladas, crescem de forma independente e espalham-se rapidamente do local inicial para outros tecidos, formando focos secundários denominados metástases. Entre as inovações aplicadas na área oncológica, está a utilização dos nanomateriais, os quais têm o intuito de melhorar o desempenho tanto em termos de biodisponibilidade quanto eficácia e segurança, devido as suas propriedades em nanoescala. O óxido de grafeno tem uma estrutura hexagonal, sendo considerado um dos materiais mais populares recentemente, tendo em vista as suas excelentes propriedades físicas, elétricas e térmicas semelhantes ao grafeno. Atualmente, novas alternativas com a utilização do óxido de grafeno vêm sendo desenvolvidas, neste sentido, este trabalho tem o objetivo de analisar as aplicações recentes do uso do óxido de grafeno para o tratamento oncológico. O estudo caracteriza-se como uma revisão bibliográfica na qual se realizou um levantamento bibliográfico entre 20152020. Os artigos selecionados limitaram-se a apenas à língua inglesa e na busca com os termos "cancer" e "graphene oxide", foram encontrados 102 artigos, sendo cinco artigos selecionados para a análise deste traba1ho. Dessa forma, verifica-se que os resultados que abordam a utilização de óxido de grafeno são promissores e demonstram-se eficazes alternativas no tratamento do câncer, no entanto, ainda necessita-se de mais estudos a fim de garantir a segurança e a sua aplicabilidade para o tratamento oncológico.

Palavras-chave: Inovações tecnologias; Quimioterapia; Nanopartículas; Tratamento oncológico.

\section{ABSTRACT}

Cancer is caused by cell transformation in the cell division process. Cancer cells are usually uncontrolled, grow independently and spread quickly from the initial site to other tissues, forming secondary foci called metastases. Among the innovations applied in the oncology area, is the use of nanomaterials, which aims to improve the performance of both in terms of bioavailability as well as effectiveness and safety, due to their nanoscale properties. Graphene oxide has a hexagonal structure, being considered one of the most popular materials recently, in view of its excellent physical, electrical and thermal properties similar to graphene. Currently, new alternatives with the use of graphene oxide have been developed, in this sense, this work aims to analyze the recent applications of the use of graphene oxide for cancer treatment. The study is characterized as a bibliographic review in which a bibliographic survey was carried out between 2015-2020. The selected

1 Trabalho de conclusão de curso de especialização em Oncologia - Santa Maria - RS.

2 Doutoranda em Nanociências - Santa Maria-RS. E-mails: lenisedeon@gmail.com

3 Orientadora. Doutora em Nanociências e Especialista em Terapia Intensiva com Ênfase em Oncologia e Controle de Infecção - Santa Maria - RS E-mail: anajuliadalcin@hotmail.com

4 Contribuidoras. Mestra e Doutoranda em Nanociências - Santa Maria - RS. E-mail: evelin_cogo@msn.com; andripinton@ gmail.com

5 Contribuidora. Mestranda em Ciências da Saúde e da Vida - Santa Maria - RS. E-mail: nara.dutra@iffarroupilha.edu.br 
articles were limited to the English language only and in the search with the term's "cancer" and "graphene oxide", 102 articles were found, where 5 articles were selected for the analysis of this work. Thus, it appears that the results that address the use of graphene oxide are promising and prove to be effective alternatives in the treatment of cancer, however, further studies are still needed in order to ensure safety and its applicability. for cancer treatment.

Keywords: Chemotherapy; Innovations technologies; Nanoparticles; Oncological treatment.

\section{INTRODUÇÃO}

O câncer é causado pela transformação celular no processo de divisão celular. Durante esse processo, o câncer começa a crescer e dividir-se mais rapidamente do que as células normais, perdendo a sua função. O material genético (DNA) de células normais que se tornam células malignas altera e as células cancerosas, geralmente, crescem de forma independente e espalham-se rapidamente do local inicial para outros tecidos, formando focos secundários chamados metástases (ARAÚJO; ROSANELLI; LORO, 2011).O processo de formação do câncer é chamado de carcinogênese ou oncogênese e comumente ocorre lentamente, podendo levar anos para que as células cancerosas proliferem e produzam tumores visíveis. O acúmulo de diferentes agentes cancerígenos ou carcinógenos é responsável pela iniciação, promoção, desenvolvimento e inibição de tumores (INCA, 2020).

As taxas de incidência e mortalidade por câncer têm aumentado em todo o mundo, em grande parte devido ao envelhecimento e crescimento populacional e às mudanças na distribuição e prevalência de fatores de risco de câncer. Os principais tipos de cânceres observados nos países em desenvolvimento estão mudando, os tipos de câncer relacionados a infecções diminuíram e os hábitos e atitudes relacionados à urbanização aumentaram como uma melhoria das condições socioeconômicas, além dos hábitos alimentares relacionados ao estilo de vida (BRAY; FERLAY; SOERJOMATARAM, 2018).

Uma das aplicações mais frequentes na área da oncologia é a utilização dos nanomateriais, que vêm sendo utilizado como uma alternativa para melhorar o desempenho de fármacos convencionais tanto em termos de biodisponibilidade quanto eficácia e segurança, bem como o desenvolvimento de novos fármacos devido às vantagens das suas propriedades em nanoescala (BREGOLI; MOVIA; GAVIGAN-IMEDIO, 2016).

Pesquisadores têm desenvolvido materiais à base de grafeno apontando sua aplicabilidade em diversas áreas de conhecimento em razão de suas características promissoras (GEIM, A.K.; NOVOSELOV, K.S, 2007). O grafeno possui propriedades únicas, as quais apresentam alta capacidade no desempenho de materiais, devido a sua condutividade elétrica e térmica, boa transparência e resistência mecânica e alta área superficial específica (SUN et. al. 2011).

Atualmente, novas alternativas com o óxido de grafeno vêm sendo desenvolvidas de acordo com sua aplicação, e uma delas é na área oncológica. Dessa forma, muitos estudos visam às novas formas de tornar os fármacos mais eficazes e mais seguros. Neste sentido, este trabalho tem como 
objetivo analisar as aplicações recentes do uso do óxido de grafeno, bem como avaliar o seu impacto e as perspectivas da sua utilização para o tratamento oncológico.

\section{OBJETIVO}

Este trabalho teve como objetivo realizar uma revisão da literatura abordando as temáticas envolvidas entre óxido de grafeno e câncer, bem como verificar o impacto dessas nanopartículas no tratamento oncológico.

\section{METODOLOGIA}

O estudo caracteriza-se como um estudo de revisão bibliográfica, no qual se realizou um levantamento bibliográfico acerca da base de dados científicos: Science Direct.

O levantamento bibliográfico foi restrito às publicações dos últimos cinco anos (2015 a 2020) e as palavras utilizadas para a busca foram: oncologia; óxido de grafeno e câncer. Os artigos selecionados limitaram-se apenas à língua inglesa e foram encontrados 596.425 artigos na primeira busca, abordando o tema oncologia. Em uma segunda busca com o termo "graphene oxide", foram encontrados 491 artigos. Em uma terceira busca com o termo "cancer", foram encontrados 102 artigos, sendo selecionados cinco artigos para a composição deste trabalho. Os artigos que foram excluídos para realização do artigo fugiram do tema proposto ou a nanopartícula utilizada não se restringia a óxido de grafeno.

\section{REFERENCIAL TEÓRICO}

\section{CÂNCER}

O câncer é uma doença caracterizada pela proliferação descontrolada de células anormais que invadem as estruturas das células adjacentes que continuamente espalham-se e espalham-se pela corrente sanguínea, vasos linfáticos ou cavidades corporais a uma certa distância (metástase) (INCA, 2020).

O aumento da morbimortalidade da doença é atribuído principalmente a aspectos como crescimento populacional, envelhecimento populacional, desenvolvimento socioeconômico e tecnológico e a industrialização de alimentos. Dessa forma, aponta-se o câncer como um problema de saúde pública (OLIVEIRA, PINHEIRO, MELO, 2011; BARROS, 2016).

Os dados mais recentes mundialmente apontam que 18 milhões de novos casos de câncer ocorreram no mundo (17 milhões sem contar os casos de câncer de pele não melanoma) e 9,6 milhões de mortes (9,5 milhões excluindo o câncer de pele não melanoma). O câncer de pulmão é o considerado o mais prevalente no mundo (2,1 milhões), seguido pelo câncer de mama (2,1 milhões), câncer 
de cólon e reto (1,8 milhão) e câncer de próstata (1,3 milhão). Para os homens, a incidência é de 9,5 milhões, sendo que se apresenta 53\% dos novos casos, e as mulheres apresentam 8,6 milhões, com $47 \%$ de novos casos. Os tipos de câncer mais comuns em homens são câncer de pulmão (14,5\%), câncer de próstata $(13,5 \%)$, câncer de cólon e reto $(10,9 \%)$, câncer de estômago $(7,2 \%)$ e câncer de fígado (6,3\%). Entre as mulheres, a maior incidência é o câncer de mama (24,2\%), cólon e reto (9,5\%), câncer de pulmão (8,4\%) e colo do útero (6,6\%) (BRAY; FERLAY; SOERJOMATARAM, 2018).

Durante o triênio 2020-2022, a estimativa é de que o Brasil terá 625.000 novos casos de câncer a cada ano. Um dos principais fatores de risco apontados é a obesidade, pois ocorre para o desenvolvimento de 11 dos 19 mais comuns tipos de câncer na população brasileira. Os fatores de risco podem ser encontrados tanto no ambiente físico, mas também nos desenvolvimentos de hábitos de vida, como, por exemplo: fumar, beber, falta de exercícios e dieta insuficiente, o que aumenta o risco de 10 tipos de doenças (INCA, 2018).

Para a execução do tratamento oncológico, é necessária uma avaliação contínua da evolução do paciente, em que precisam ser analisadas as características físicas do paciente e os aspectos do tumor. Sendo assim, analisa-se qual tratamento será adequado, seja ele por meio da quimioterapia, radioterapia, cirúrgico ou por hormonioterapia, dependendo do estado do paciente, bem como o tipo de câncer (SILVA, 2006). Para analisar a eficácia terapêutica, faz-se através de parâmetros, em que são analisados: diminuição do tumor, intervalo livre da doença e toxicidade. Diante disso, os resultados devem ser avaliados trazendo as limitações físicas e psicológicas a cada paciente em seu estágio da doença (INCA, 2018).

\section{NANOTECNOLOGIA}

A necessidade de novas tecnologias para a utilização de materiais existentes vem desde o início da revolução científica, em que o conceito emergente sobre nanociência e nanotecnologia mantém uma essência, a qual é conhecida por ter uma escala em tamanho menor (DURÁN; AZEVEDO, 2002).

A importância dos materiais nanométricos está relacionada diretamente com algumas propriedades físicas e químicas que podem ser alteradas na nanoescala. E correlacionada com as leis da física clássica, em que são corroboradas e atuam de acordo com as leis da física quântica (SOARES; ALMEIDA; SILVA, 2017). A nanotecnologia é conhecida por estar na escala nanométrica $\left(10^{-9} \mathrm{~m}=1 \mathrm{~nm}\right)$, com comprimentos que não ultrapassem 100 nanômetros em pelo menos uma direção (USKOKOVIĆ, 2007).

O prefixo "nano" originou-se no grego e significa um bilionésimo de metro (0,000000001 m), que é expresso por uma unidade de medida. Portanto, o estudo de estruturas atômicas e moleculares com dimensões em nanoescala é denominado nanociência. Por outro lado, a manipulação e 
aplicações industriais, dessas nanoestruturas são chamadas de nanotecnologia (SCHULZ, 2005). Os sistemas em nanoescala podem ocorrer de duas maneiras diferentes: sendo ela de baixo para cima (bottom-up) ou de cima para baixo (top-down). Para o método bottom-up, átomos e moléculas são quimicamente conectados de uma forma que agregados mantêm-se auto-organizados, mais conhecidos como "blocos de construção", que são usados em nanodispositivos. No método top-dow, o material é proporcionalmente reduzido até atingir o tamanho nano, ou seja, os blocos de construção são continuamente removidos da estrutura macro, para obter-se um sistema em escala nano (IQBAL; PREECE; MENDES, 2012).

Materiais ou nanomateriais com nanoestruturas em, pelo menos, uma de suas dimensões têm demonstrado propriedades satisfatórias. Suas aplicações têm sido estabelecidas especialmente na área médica e farmacêutica, com o desenvolvimento de novas formas farmacêuticas ou contribuindo para a melhoria dos fármacos convencionais (WENG et al., 2017; MALI; BATHE, 2015; PUTHETI et al., 2008).

Com a utilização da nanotecnologia, pode-se reduzir doses e a incidência de efeitos adversos, além de promover a liberação prolongada do fármaco no seu local de ação, aumentar o intervalo entre as doses aplicadas e a obtenção de uma melhor eficácia devido ao direcionamento do fármaco aos tecidos doentes com a proteção simultânea dos tecidos saudáveis. Esses sistemas de liberação de fármacos podem facilmente interagir com biomoléculas localizadas na superfície das células ou no seu interior (MALI; BATHE, 2015; BOISSEAU; LOUBATO, 2011; ASLAM, 2008).

\section{ÓXIDO DE GRAFENO}

Óxido de grafeno é quimicamente análogo ao grafite oxidado, pois é constituído por estruturas com uma camada de grafeno funcionalizado ligadas a grupos epóxi, hidroxila, carboxílicos e carbonila (EDWARDS; COLEMAN, 2013). Porém, dependendo da quantidade, do tipo e da localização dos grupos funcionais, as propriedades do óxido de grafeno podem variar, ocasionando no tempo de oxidação e temperatura dos agentes oxidantes (SHAMAILA, 2016).

A sua estrutura é uma hexagonal, sendo considerado um dos materiais mais populares recentemente, tendo em vista as suas excelentes propriedades físicas, elétricas e térmicas semelhantes ao grafeno (DUYGU; BOCCACCINI, 2017). Porém, durante sua oxigenação, as ligações $\pi$ reagem com os grupos funcionais carboxilas e hidroxilas, tornando em carbonos sp3. (ALMEIDA; DOS SANTOS; STAUDT, 2019). Por possuir carbonos sp3 na sua estrutura, torna-se um excelente material isolante, pois aumenta o valor de gap entre a banda de valência e condução (PUMERA, 2010).

Para obter a síntese do óxido de grafeno, faz-se através do método de Hummers, o que é feito através da oxidação do grafite, gerando grupos hidrofílicos na superfície. O processo é dividido em duas etapas: oxidação e a esfoliação (COMPTON; NGUYEN, 2010). 
Atualmente, novas alternativas com o óxido de grafeno vêm sendo desenvolvidas na área oncológica. Entre os estudos em desenvolvimento, Dos Santos e colaboradores (2018) relatam que a utilização do óxido de grafeno pode ser usado como um agente fototérmico em fototerapia, induzindo hipertermia e intensificando a quimioterapia.

Outro estudo foi desenvolvido por Lu e colaboradores (2017), utilizando óxido de grafeno para terapia fototérmica e quimioterapia combinada com a doxorrubicina para o tratamento do câncer colorretal subcutâneo em modelos de camundongos. O estudo mostrou que o óxido de grafeno magnético modificado com polietileno e cetuximabe foi usado para entrega dupla-alvo de um medicamento anticâncer doxorrubicina, que mostrou citotoxicidade aumentada para CT-26 in vitro e inibiu a propagação do tumor in vivo. Os efeitos antitumorais podem ser aumentados usando um laser próximo ao infravermelho para terapia fototérmica in vitro e in vivo.

Aliado a isso, segundo Shao (2017), o óxido de grafeno pode ser utilizado como um nanocarreador, pois tem grandes vantagens como: grande área de superfície específica, excelente eficiência de conversão fototérmica, fácil superfície de modificação.

Em um estudo feito por Fiorillo e colaboradores (2015), os resultados mostraram que o tratamento com nano-flocos de óxido de grafeno inibiu a formação da esfera do tumor em seis tipos de câncer linhas celulares: câncer de mama, ovário, próstata, pulmão, pâncreas e cérebro. Além disso, mostrou eficácia em reduzir o número de células troncos cancerosas.

Zhou e colaboradores (2014) relataram que o óxido de grafeno modificado com o polietilenoglicol inibe as propriedades invasivas de linhagens de células metastáticas de câncer de mama através da inativação da síntese de ATP (adenosina trifosfato), reduzindo a expressão de genes envolvidos no metabolismo energético, diminuindo a fosforilação oxidativa mitocondrial. Também observaram que não apresentou toxicidade para células epiteliais mamarias normais e, diante disso, pode ser utilizado como carreador de drogas e terapia fototérmica.

Em outro estudo, Robinson e colaboradores (2010) analisaram o estudo com folhas de óxido de grafeno reduzido (nano-rGO) com citrato de tamoxifeno. O tamoxifeno tem a capacidade de inibir o efeito do estrogênio, o qual é necessário para a propagação de certos cânceres de mama. Diante disso, a nano-rGO preparada por redução química, pode aumentar a absorbância do infravermelho próximo (NIR) em> 5 vezes. Devido a presença de grupos funcionais na superfície de rGO, um surfactante foi revestido de forma não covalente em nano-rGO para biocompatibilidade em soluções tampão biológicas. Óxido de grafeno na forma reduzida modificada com citrato de 
Tamoxifeno tem como objetivo ser um agente fototérmico com relação absorbância de luz NIR do que outros materiais nano.

\section{RESULTADOS E DISCUSSÃO}

Na primeira busca por "oncology", foram encontrados 596.425 artigos, sendo filtrados os anos de 2015-2020. Em uma segunda busca com o termo "graphene oxide", foram encontrados 491 artigos. Em uma terceira busca com o termo "cancer", foram encontrados 102 artigos, quando foram selecionados cinco para a composição da amostra dessa revisão como pode ser observado no fluxograma a seguir da Figura 1.

Figura 1 - Fluxograma referente aos artigos encontrados na base virtual.

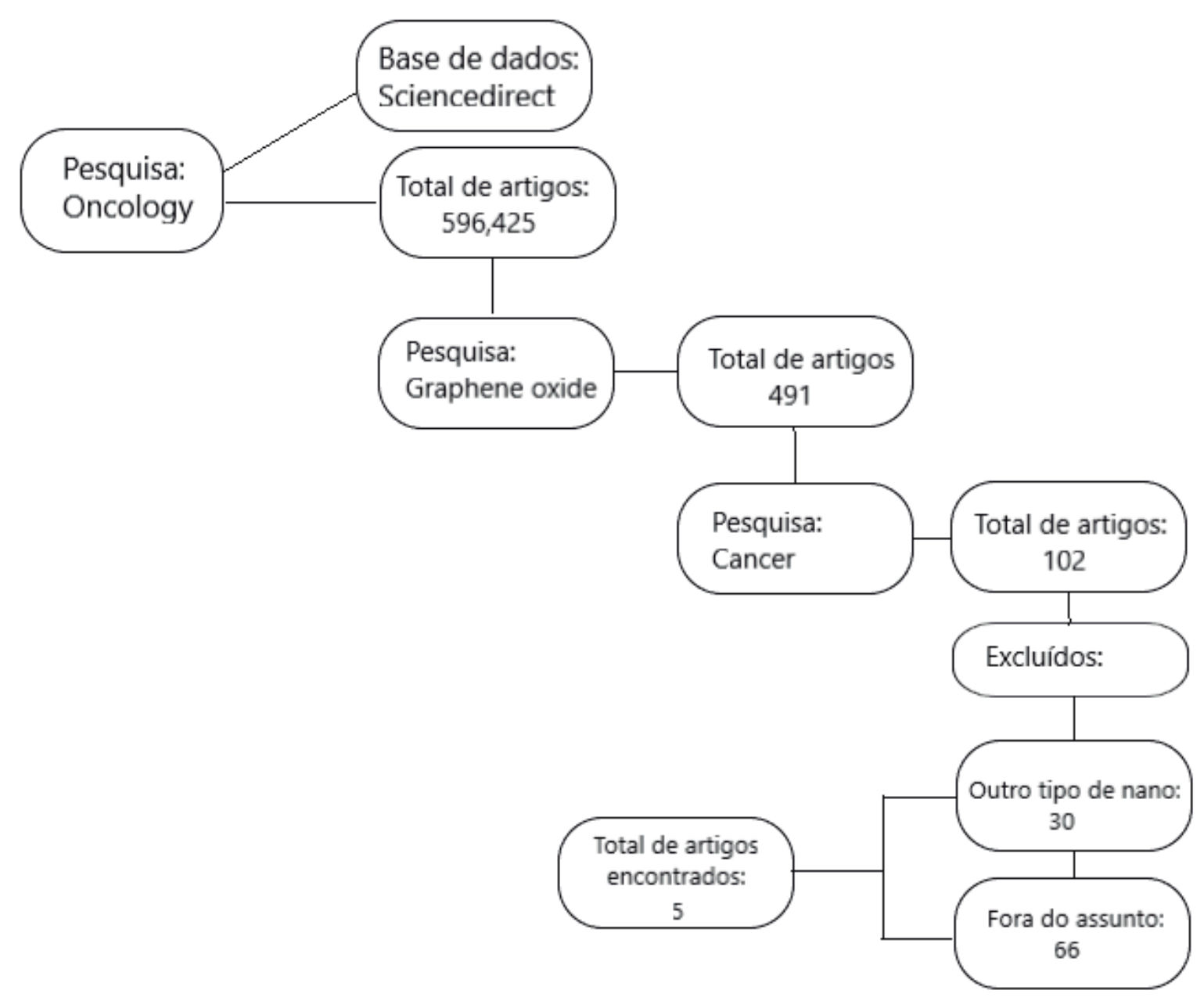

Fonte: Construção da autora.

Atualmente, não existem muitos artigos que abordem a aplicação no tratamento oncológico utilizando o óxido de grafeno e, após a leitura crítica dos artigos selecionados, restaram apenas cinco artigos apresentados conforme descrito na Tabela 1. 
Tabela 1 - Artigos acerca da aplicação no tratamento oncológico utilizando óxido de grafeno no período 2015-2020.

\begin{tabular}{|c|c|c|c|}
\hline $\begin{array}{c}\text { Forma do } \\
\text { óxido de grafeno }\end{array}$ & Objetivo do estudo & Conclusão & Referência \\
\hline $\begin{array}{l}\text { Nanocompósito à } \\
\text { base de óxido de } \\
\text { grafeno }\end{array}$ & $\begin{array}{l}\text { Fabricar nanocompósitos de óxido de } \\
\text { grafeno com o objetivo de modificar a } \\
\text { entrega do medicamento 5-fluorouracil, } \\
\text { o qual é um fluoreto de pirimidina e inibe } \\
\text { o crescimento de células cancerosas via } \\
\text { inibição da timidilato sintase, que afeta a } \\
\text { biossíntese de DNA para o tratamento do } \\
\text { câncer colorretal. }\end{array}$ & $\begin{array}{l}\text { Resultados in vitro e in vivo confirmaram } \\
\text { que os nanocompósitos serviram como um } \\
\text { eficiente transportador de droga para célu- } \\
\text { las cancerosas com superexpressão do re- } \\
\text { ceptor de fator de crescimento epidérmico } \\
\text { (EGFR) ou tumor local. E podem melhorar } \\
\text { notavelmente o efeito da quimioterapia } \\
\text { juntamente com terapia fototérmica }\end{array}$ & $\begin{array}{l}\text { ZHICHAO QIU } \\
\text { et al. (2020) }\end{array}$ \\
\hline $\begin{array}{l}\text { Nano-flocos de } \\
\text { óxido de grafeno } \\
\text { (GONFs) }\end{array}$ & $\begin{array}{l}\text { Investigar a viabilidade do uso de óxido } \\
\text { de grafeno durante a radioterapia para } \\
\text { inibir sinergicamente as células-tronco } \\
\text { cancerosas e reduzir o risco de metástase } \\
\text { e recorrência do câncer }\end{array}$ & $\begin{array}{l}\text { Neste trabalho, uma concentração de } \\
100 \mu \mathrm{g} \text { / mL de GONFs demonstrou } \\
\text { ser tóxico para células A549 (uma linha } \\
\text { de células de câncer de pulmão) e não } \\
\text { tóxico para células HUVEC (uma linha de } \\
\text { células de tecido normal). Após a difusão, } \\
\text { apenas GONFs de tamanho } 50 \text { e } 200 \mathrm{~nm} \\
\text { entregou a concentração efetiva ( } 50 \mu \mathrm{g} \text { / } \\
\text { mL) em } 14 \text { ou } 21 \text { dias em todo o volume } \\
\text { do tumor. Combinando GONFs com } \\
\text { radiação, a morte das células cancerosas } \\
\text { pode ser aumentada. Os autores mencio- } \\
\text { nam que se necessita de mais estudos para } \\
\text { desenvolver o uso de GONFs durante a } \\
\text { radioterapia guiada por imagem }\end{array}$ & $\begin{array}{l}\text { DOLLA } \\
\text { TOOMEH et al. } \\
(2018)\end{array}$ \\
\hline $\begin{array}{l}\text { Filme de óxido de } \\
\text { grafeno reduzido }\end{array}$ & $\begin{array}{l}\text { Foi desenvolvido um aptasensor flexível } \\
\text { e ultrassensível baseado em nanotubos } \\
\text { de carbono de paredes múltiplas carbo- } \\
\text { xilados/ transistor de efeito de campo } \\
\text { baseado em óxido de grafeno reduzido } \\
\text { para a detecção do marcador do antígeno } \\
\text { de câncer de ovário (CA125). }\end{array}$ & $\begin{array}{l}\text { Com base nos desempenhos de detecção } \\
\text { do aptasensor, considera-se aplicado } \\
\text { com sucesso para detectar o marcador de } \\
\text { antígeno CA125 em amostras de soro hu- } \\
\text { mano. Além disso, o aptasensor também } \\
\text { exibiu excelente flexibilidade mecânica, } \\
\text { alta reprodutibilidade, estabilidade de lon- } \\
\text { ga duração, baixo custo e fácil fabricação. }\end{array}$ & $\begin{array}{l}\text { MANSOURI } \\
\text { MAJD; } \\
\text { ABDOLLAH } \\
\text { SALIMI. (2018) }\end{array}$ \\
\hline $\begin{array}{l}\text { Óxido de grafeno } \\
\text { reduzido }\end{array}$ & $\begin{array}{l}\text { Avaliar a citotoxicidade do óxido de gra- } \\
\text { feno reduzido contra as linhas de células } \\
\text { de câncer cervical (HeLa). }\end{array}$ & $\begin{array}{l}\text { A citotoxicidade in vitro do óxido de grafe- } \\
\text { no reduzido mostrou ser menos tóxica con- } \\
\text { tra as linhas celulares HeLa. Além disso, a } \\
\text { natureza menos tóxica do óxido de grafeno } \\
\text { reduzido funcionalizado com pirogalol } \\
\text { preparado para a aplicação de materiais de } \\
\text { grafeno para a fototerapia térmica. }\end{array}$ & $\begin{array}{l}\text { LAN LUO et al. } \\
\text { (2017) }\end{array}$ \\
\hline $\begin{array}{l}\text { Óxido de } \\
\text { grafeno reduzido } \\
\text { modificado com } \\
\text { citrato de } \\
\text { Tamoxifeno } \\
\text { (nano-rGO) }\end{array}$ & $\begin{array}{l}\text { Desenvolver uma abordagem simpli- } \\
\text { ficada para a fabricação de óxido de } \\
\text { grafeno reduzido modificado com citrato } \\
\text { de Tamoxifeno (nano-rGO) com mais } \\
\text { estabilidade e baixa citotoxicidade para o } \\
\text { tratamento de câncer de mama. }\end{array}$ & $\begin{array}{l}\text { O nano-rGO carregado com tamoxifeno } \\
\text { apresentou tanto estabilidade significativa } \\
\text { como citotoxicidade. Além disso, também } \\
\text { poderia ser empregado para fototerapia no } \\
\text { tratamento de câncer de mama. }\end{array}$ & $\begin{array}{l}\text { YU-JIN ZHANG } \\
\text { et al. (2017) }\end{array}$ \\
\hline
\end{tabular}

Fonte: Construção da autora. 
Foram analisados cinco artigos relacionando o tema óxido de grafeno e câncer. Destes, dois artigos avaliaram a eficácia do quimioterápico juntamente com terapia fototérmica (ZHICHAO QIU et al., 2020; YU-JIN ZHANG et al.,2017).

A terapia fototérmica é utilizada para acelerar a morte de células tumorais através do acúmulo de calor em tumores por meio de um material de conversão fototérmico no infravermelho próximo de (700-1000 nm) (LI et al., 2012). A utilização do óxido de grafeno como agente fototérmico é relatada pela indução da hipertermia, aumentando a intensidade e sensibilidade na quimioterapia no tratamento do câncer. Neste sentido, o óxido de grafeno tem um grande potencial para ser um nanocarreador, pois tem como vantagem a grande área de superfície específica, excelente eficácia na conversão fototérmica e fácil superfície modificada (SHAO et al., 2017).

Um nanocompósito com oxido de grafeno foi analisado para alcançar a terapia fototérmica e quimioterapia sinérgica do câncer colorretal subcutâneo. O óxido de grafeno foi modificado para entregar o medicamento 5-fluorouracil (5-FU), sendo que esse medicamento atua na função de inibir o crescimento das células cancerosas através da inibição do timidilato sintase (SHARMA et al., 2017).

Lan Lou e colaboradores (2017) avaliaram a citotoxicidade através do ensaio de MTT (brometo de 3-[4,5-dimetil-tiazol-2-il]-2,5-difeniltetrazólio) para avaliar a viabilidade celular após o tratamento com óxido de grafeno e óxido de grafeno reduzido. Os resultados demonstraram um declínio na viabilidade das células HeLa (linhagem celular imortal cultivada em laboratórios) com aumento na concentração de 10 a 100 ppm para oxido de grafeno e óxido de grafeno reduzido. Os resultados in vitro do óxido de grafeno reduzido apontaram que a citotoxicidade em relação às células HeLa é dependente da dose.

Outro artigo avaliou a especificidade na célula tumoral e identificou que os nano-flocos de óxido de grafeno (GONFs) puderam ser entregues de forma sustentável in situ na radioterapia. A entrega in situ ajudaria a superar barreiras fisiológicas, como membrana endotelial vascular e matriz extracelular, que impedem a entrega de concentrações suficientemente potentes de GONFs ao tumor. Os autores relataram que a entrega sustentada ao longo da duração da radioterapia seria mais desejável em comparação com injeções individuais repetidas. A entrega sustentada pode ser personalizada por vários parâmetros de design como a concentração de carga útil, tipo ou peso de polímero e o tamanho de GONFs (DOLLA TOOMEH et al., 2018)

O quinto artigo foi realizado por Mansouri Majd e colaboradores (2018), que avaliaram a possibilidade do desenvolvimento de um sensor que detectou o antígeno de câncer de ovário (CA125). O uso de dispositivos foi aprimorado com o uso de nanoestruturas, como nanofios de silício, nanotubos de carbono e grafeno. O grafeno não só tem excelente mobilidade de elétrons, como uma ótima condutividade térmica, alta resistência mecânica e grande relação superfície-volume. (GEIM, A.K.; NOVOSELOV, K.S, 2007). Essas características tornaram o grafeno um material atraente de transdutores. Nesse estudo, realizou-se uma metodologia de um aptasensor do tipo FET (transistor de efeito de campo) flexível e demonstrou sua capacidade para detecção seletiva sensível de detectar o antígeno CA125. 


\section{CONSIDERAÇÕES FINAIS}

Esta revisão teve como objetivo analisar artigos encontrados na literatura referente à temática câncer e óxido de grafeno (GO). Foi possível observar que atualmente existem poucos relatos de publicações abordando esse tema. Entre os estudos promissores, está a avaliação da eficácia de quimioterápicos juntamente com terapia fototérmica utilizando GO para induzir hipertermia e aumentar a sensibilidade da quimioterapia no tratamento do câncer, bem como a utilização de nano-flocos de GO para avaliar a especificidade na célula tumoral na entrega de forma sustentável in situ na radioterapia, assim como foi encontrado um estudo referente ao desenvolvimento de um sensor para detectar o antígeno de câncer de ovário, utilizando nanoestruturas com silício, nanotubos de carbono e grafeno. Sendo assim, tendo em vista que, apesar das escassas publicações envolvendo o tema citado, os resultados existentes são promissores e revelam um potencial a ser explorado com a utilização de óxido de grafeno no tratamento e ou detecção do câncer.

\section{REFERÊNCIAS}

ALMEIDA, A. F; DOS SANTOS, G. S. F; STAUDT. J; BARICATTI, R. A., Síntese e caracterização de óxido de grafeno pelo método de oxidação química do grafite visando suas aplicações em adsorção de resíduos. IBEAS - instituto brasileiro de estudos ambientais., Foz do Iguaçu, 2019.

ARAÚJO A. D.; ROSANELLI C. L. P. S.; LORO M. M.; STUMM E. M. F.; KOLANKIEWICZ A. C. B. Complicações em pacientes oncológicos decorrentes do uso de cateter de longa permanência. Rev enferm UFPE online. v. 5, n. 4, p. 916-23, 2011.

ASLAM, S. Effect of antibacterials on biofilms. American Journal of Infection Control. v. 36, n. 10, p. 9-11, 2008.

BARROS HCS. Aspectos epidemiológicos da mortalidade por câncer de mama masculina no Brasil, no período de 1996 a 2013. Dissertação de Mestrado, Maceió (AL), 2016.

BOISSEAU, P; LOUBATON B; Nanomedicine: nanotechnology in medicine. Comptes Rendus Physique. n. 12, p. 620-636, 2011.

BRAY F, FERLAY J, SOERJOMATARAM I, SIEGEL RL, TORRE LA, Jemal A. Global cancer statistics 2018: GLOBOCAN estimates of incidence and mortality worldwide for 36 cancers in 185 countries. CA Cancer J Clin, v. 68, n. 6, p. 394-424, Nov. 2018. 
BREGOLI, L.; MOVIA, D.; GAVIGAN-IMEDIO, J. D; LYSAGHT, J.; REYNOLDS, J.; PRINA-MELLO, A.; Nanomedicine applied to translational oncology: A future perspective on cancer treatment. Nanomedicine: Nanotechnology, Biology, and medicine. v. 12, p. 81-103, 2016.

COMPTON, O.C.; NGUYEN, S.T. Graphene oxide, highly reduced graphene oxide, and graphene: Versatile building blocks for carbon-based materials. Small, v. 6, n. 6, p. 711-723, 2010.

DOLLA TOOMEHA, SHERIF M. GADOUE, SAYEDA YASMIN-KARIM, MANJRI SINGH, RISHI SHANKER, SURINDER PAL SINGH, RAJIV KUMAR, ERNO SAJO, WILFRED NGWA, Minimizing the potential of cancer recurrence and metastasis by the use of graphene oxide nano-flakes released from smart fiducials during imageguided radiation therapy, Physica Medica, 2018.

DOS SANTOS MSC, GOUVÊA AL, DE MOURA LD, PATERNO LG, DE SOUZA PEN, BASTOS AP, DAMASCENO EAM, VEIGA-SOUZA FH, DE AZEVEDO RB, BÁO SN. Nanographene oxide-methylene blue as phototherapies platform for breast tumor ablation and metastasis prevention in a syngeneic orthotopic murine model. J Nanobiotechnology. 2018 doi: 10.1186/s12951-018-0333-6.

DURÁN, N. ; AZEVEDO, M. M. Rede de pesquisa em nanobiotecnologia. 2002. Disponível em: https://bit.ly/3iMLaNr. Acesso em: 12 de outubro de 2020.

DUYGU EGE, A. R. K., ALDO R. BOCCACCINI. “Graphene Oxide/PolymerBased Biomaterials.” Advanced Engineering Materials, 2017.

EDWARDS, R. S.; COLEMAN, K. S. Graphene synthesis: relationship to applications. Nanoscale, v. 5, n. 1, p. 38-51, 2013.

FIORILLO M, VERRE AF, ILIUT M, PEIRIS-PAGES M, OZSVARI B, GANDARA R, Graphene oxide selectively targets cancer stem cells, across multiple tumor types: implications for non-toxic cancer treatment, via "differentiation-based nano-therapy". v. 6, p. 3353-6, 2015.

GEIM, A. K.; NOVOSELOV, K. S. The rise of graphene. Nature Materals, v. 6, p .183-191, 2007. IQBAL, P. ; PREECE, J. A.; MENDES, P. M. Nanotechnology: The "Top-Down" and "Bottom-Up" Approaches. Supramolecular Chemistry, p. 1-14, John Wiley \& Sons, Ltd; 2012. 
INCA INSTITUTO NACIONAL DE CANCER. 2020. Disponível em: https://bit.ly/35yFBdz. Acesso em: 21 dez. 2020.

INSTITUTO NACIONAL DO CANCER - INCA (2018). Prevenção e fatores de Risco. Disponível em: https://bit.ly/2Uf6AZo. Acesso em: 2 set. 2020.

LAN LUO, LINA XU, HAIBO ZHAO, Biosynthesis of reduced graphene oxide and its in-vitro cytotoxicity against cervical cancer (HeLa) cell lines, Materials Science \& Engineering C, 2017.

LI M, YANG X, REN J, QU K, QU X, Using Graphene Oxide High Near-Infrared Absorbance for Photothermal Treatment of Alzheimer's Disease, Adavanced Materials, p. 1722-1728, 2012.

LU Y. J., LIN P. Y., HUANG P. H., KUO C. Y., SHALUMON K. T., CHEN M. Y., CHEN J. P. , Magnetic Graphene Oxide for Dual Targeted Delivery of Doxorubicin and Photothermal Therapy, Nanomaterials, p. 193-212, 2018.

MALI A. D.; BATHE R. S. Updated review on nanoparticles as drug delivery systems. International Journal of Analytical. Pharmaceutical and Biomedical Sciences. v. 8, n. 9, p. 18-34, 2015.

MANSOURI MAJD S, SALIMI A. Ultrasensitive flexible FET-type aptasensor for CA 125 cancer marker detection based on carboxylated multiwalled carbon nanotubes immobilized onto reduced graphene oxide film. Analytica Chimica Acta. 2018

MCNAUGHT, A. D.; WILKINSON, A.; Publications, B. S. Graphene layer. Disponível em: www. goldbook.iupac.org. Acesso em: 3 nov. 2020.

OLIVEIRA EXG, MELO EC, PINHEIRO RS, NORONHA CP, CARVALHO MS. Acesso à assistência oncológica: mapeamento dos fluxos origem-destino das internações e dos atendimentos ambulatoriais. O caso do câncer de mama. Cadernos de Saúde Pública. p. 317-326, 2011.

PUMERA, M. Graphene-based nanomaterials and their electrochemistry., Chem. Soc. Rev. 39, p. 4146-4157, 2010.

PUTHETI, R. R.; OKIGBO, R. N. ; MADHUSOODHAN, S. and CHAVANPATIL, S. Nanotechnology importance in the pharmaceutical industry. African Journal of Pure and Applied Chemistry. v. 2, n. 3, p. 27-31, 2008. 
ROBINSON, J. T, PERKINS, F. K, SNOW, E. S, WEI, Z., SHEEHAN, P. E, Reduced graphene oxide molecular sensors, Nano Lett. 8, 2008.

ZHICHAO QIUA, JING HUB, ZIWEI LIC, XIAOXUE YANGC, JUN HUC, QINGJUN YOUA, SHUO BAID, YONG MAOA, DONG HUAA, JIAN YIN. Graphene oxide-based nanocomposite enabled highly efficient targeted synergistic therapy for colorectal câncer, Colloids and Surfaces A, 2020.

SHAO. L.H, ZHANG.R. R, LU.J. Q, ZHAO.C. Y, DENG.X. W, WU. Y, Mesoporous Silica Coated Polydopamine Functionalized Reduced Graphene Oxide for Synergistic Targeted Chemo-Photothermal Therapy Appl. Mater. Interfaces v. 9, p. 1226-1236, 2017.

SHAMAILA, S.; SAJJAD, A. K. L.; IQBAL, A. Modifications in development of graphene oxide synthetic routes. Chemical Engineering Journal, v. 294, p. 458-477, 2016.

SHARMAA A, KAURA A. JAINA K.U, RAMESH CHANDRA R, MADAN J, Stealth recombinant human serum albumin nanoparticles conjugating 5-fluorouracil augmented drug delivery and cytotoxicity in human colon cancer, HT-29 cells. Author links open overlay panel, Colloids and Surfaces B: Biointerfaces, v. 155, p. 200-208, 2017.

SILVA DA, N. P. M. Síndrome da anorexia-caquexia em portadores de câncer. Revista Brasileira de Cancerologia, v. 52, n. 1, p. 59-77, 2006.

SOARES, F. G.; ALMEIDA, C. A. de; SILVA, J. R. N. da. A física do impossível: nanotec-nologia, metamateriais e invisibilidade na sala de aula. In: SIMPÓSIO NACIONAL DE ENSINO DE FÍSICA, 22, 2017, São Carlos. Anais... São Carlos: 2017. p. 1-8. Disponível em: https://bit.ly/3gCvB9G. Acesso em: 27 jun. 2020.

SCHULZ, P. A. B. O que é nanociência e para que serve a nanotecnologia. Física na Escola, São Paulo, v. 6, n. 1, p. 58-62, jan. 2005. Disponível em: https://bit.ly/2TKlk1U. Acesso em: 15 ago. 2020.

SUN, Y., WU, Q., SHI, G. Graphene based new energy materials. Energy \& Environmental Science, 4, p. 1113-1132, 2011.

USKOKOVIĆ, V. Nanotechnologies: What we do not know. Technology in Society v. 29, n. 1, p. 43-61, 2007. 
WENG, Y.; LIU, J.; JIU, S.; GUO, W.; LIONG, X.; HU, Z. Nanotechnology-based strategies for treatment of ocular disease. Acta Pharmaceutica Sinica B. v. 7, n. 3, p. 281-291, 2017.

ZHOU T, ZHANG B, WEI P, DU Y, ZHOU H, YU M, Energy metabolism analysis reveals the mechanism of inhibition of breast cancer cell metastasis by PEG-modified graphene oxide nanosheets. Biomaterials; v. 35, p. 9833-9843, 2014.

YU-JIN ZHANG, BAO-AN LI, ZHAO-YUAN LI, NING XIA, HAI-YING YU, YA-ZHI ZHANG, Synthesis and characterization of Tamoxifen citrate modified reduced graphene oxide nano sheets for breast cancer therapy, Journal of Photochemistry \& Photobiology, B: Biology, 2017. 